\title{
A Rare Cause of Bowel Obstruction
}

Fezzeh Elyasinia ${ }^{1}$, Ahmadreza Soroush ${ }^{1}$, Ehsan Sadeghian 1,*, Nima Taghizadeh ${ }^{1}$, Maryam Mahdavidoust ${ }^{1}$, Reza Parsaei ${ }^{1}$, Mohammad Javad Bagheri ${ }^{1}$

1. Department of Surgery, Shariati Hospital, Tehran University of Medical Sciences, Tehran, Iran

\section{* Corresponding Author:}

Ehsan Sadeghian, MD

Shariati Hospital, Jalal-e-Al-e-Ahmad Hwy, Tehran, Iran

Tel: + 98218490

Fax: + 982188633039

Email: Ehsan810@yahoo.com

Received: 28 Nov. 2019

Accepted: 26 Feb. 2020
Please cite this paper as:

Elyasinia F, Soroush AR, Sadeghian E, Taghizadeh N, Mahdavidoust M , Parsaei R, Bagheri MJ. A Rare Cause of Bowel Obstruction. Middle East J Dig Dis 2020;12:133-135. doi: $10.34172 /$ mejdd.2020.175.

A 26-year-old female patient was admitted to our hospital, complaining of obstipation, nausea, and vomiting for the past 3 days. She had no significant medical and clinical history. The patient had no history of previous abdominal operations or medication use.

Distension of the abdomen was the only finding on clinical examination. Plain abdominal radiography showed air-fluid levels and abdominal computed tomography (CT) showed the clustered terminal ileum and cecum, with dilatation at the proximal parts (figure 1). The findings of laboratory tests were within normal limits. Exploratory laparotomy was performed.

At laparotomy, we found that loops of ileum and cecum were trapped in a fibrotic sac with a diameter of nearly $10 \mathrm{~cm}$ (figure 2).

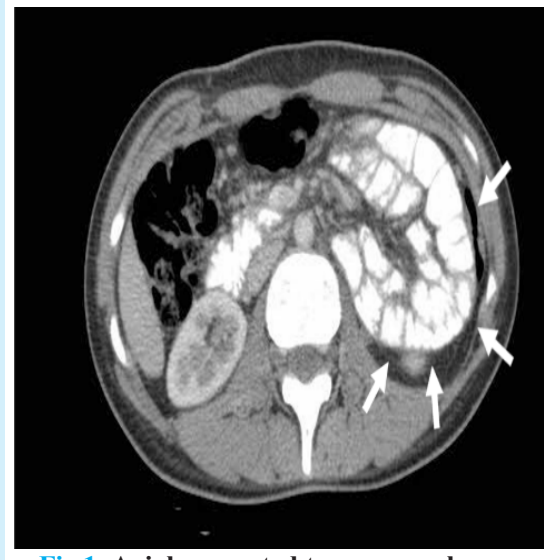

Fig.1: Axial computed tomogram shows the clustering of small bowel loops (arrows).

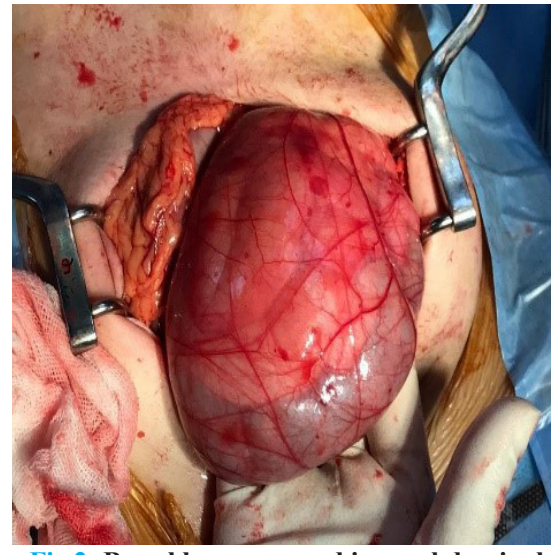

Fig.2: Bowel loops encased in an abdominal cocoon.

\section{What is your diagnosis?}

\section{Answer:}

Abdominal cocoon or sclerosing encapsulating peritonitis

Abdominal cocoon or sclerosing encapsulating peritonitis refers to the presence of a thick fibrous membrane inside the peritoneal cavity. It was first reported by Foo in $1978 .{ }^{1}$ It is usually associated with conditions that cause intraperitoneal inflammation. ${ }^{2}$ In some cases, no etiology is found and the 


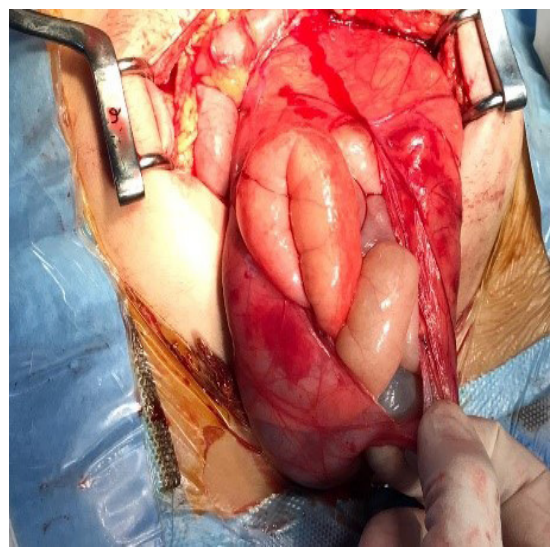

Fig.3: Incising fibrous membrane

abdominal cocoon is considered as primary or idiopathic. ${ }^{3}$ Secondary abdominal cocoon is associated with conditions including chronic ambulatory peritoneal dialysis (CAPD), peritoneal tuberculosis, systemic lupus erythematosus, and various intraperitoneal shunts. ${ }^{4}$ Etiology of primary abdominal cocoon is not completely understood but subtle viral peritonitis, immunological reactions or gynecological infections have been postulated as probable causes. ${ }^{4}$ Symptoms and physical findings of the abdominal cocoon are non-specific and suggestive of acute obstruction or chronic abdominal symptoms. Chronic symptoms include pain, nausea, vomiting, weight loss, and loss of appetite. ${ }^{3}$ Cocoon may cause symptoms by encasement of bowel loops. It presents as either acute bowel obstruction or chronic symptoms of abdominal pain, early satiety, and weight loss. ${ }^{3}$ A palpable mass of encased bowels may be found on physical examination. ${ }^{5}$ Plain abdominal imaging is of little help in differentiating abdominal cocoon from other abdominal pathologies. A contrast study showing a congregated bowel loop suggests diagnosis but is not present in most of the cases. ${ }^{4} \mathrm{CT}$ shows the local clustering of bowel loops. ${ }^{3}$ A fibrous membrane that encases bowel loops also can be detected in CT. ${ }^{6}$ Some authors defined "Cauliflower sign", the broad head formed by the bowel loops with a narrow mesenteric apex, as a feature of abdominal cocoon. ${ }^{7}$ In the report of 25 cases of the abdominal cocoon, Gorsi and colleagues have found that "Cauliflower sign" was present in 94\% of acute obstructions caused by the abdominal cocoon. Another sign is dilatation of the $2 \mathrm{nd}$ and $3 \mathrm{rd}$ parts of the duodenum with encasement of distal duodenum and jejunal loops. This is called "Bottle gourd sign" and is reported

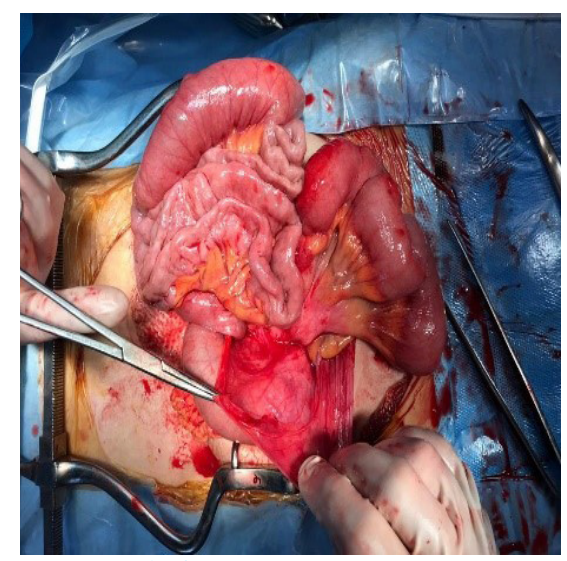

Fig.4: Released bowel loops

to be present in $50 \%$ of bowel obstructions caused by abdominal cocoon. ${ }^{3,8}$ Other features that are found in abdominal cocoon include peritoneal enhancement and thickening, adhesion of bowel loops, and fluid loculation. ${ }^{3}$ Other authors have reported high specificity and sensitivity of CT for the diagnosis of abdominal cocoon. ${ }^{9}$ In spite of suggestive features in $\mathrm{CT}$, most of the cases, similar to ours, are not diagnosed before surgery. ${ }^{10}$ Small bowel obstruction in the absence of previous surgical history is suggestive of internal herniation. This condition prompts surgical exploration and the exact diagnosis of obstruction before surgery is not necessary. Surgical treatment involves excising the fibrous membrane and releasing encapsulated bowels. This has been performed successfully in most abdominal cocoon cases. In our patient, the fibrous membrane was incised and bowel segments were released (figures 3,4). Since the bowels were viable, no resection was needed during the operation. The patient's postoperative course was uneventful and she was discharged on the fifth postoperative day.

Laparoscopic surgery has also been performed for abdominal cocoon. ${ }^{11,12}$ The decision of performing laparoscopy in case of bowel obstruction must be individualized based on the patient's condition and surgeon's expertise. In some patients with active inflammation and severe adhesions in the abdomen, surgery may not be feasible due to the high risk of iatrogenic bowel perforation. This usually happens in some cases of abdominal tuberculosis. ${ }^{13,14} \mathrm{Con}$ servative management with low residue diet and medical therapy for tuberculosis have been used in such cases and in patients with chronic symptoms that have been diagnosed by imaging. Medical therapy has been successful in 
resolving symptoms. However, follow-up imaging reveals persistent cocoon in most of the patients. ${ }^{3,13}$

An abdominal cocoon should be beard in mind when a cluster of bowel loops is seen in the computed tomogram of a patient who presents with bowel obstruction.

\section{ETHICAL APPROVAL}

There is nothing to be declared.

\section{CONFLICT OF INTEREST}

The authors declare no conflict of interest related to this work.

\section{REFERENCES}

1. Foo KT, Ng KC, Rauff A, Foong WC, Sinniah R. Unusual small intestinal obstruction in adolescent girls: the abdominal cocoon. Br J Surg 1978; 65:427e30. doi: $10.1002 /$ bjs. 1800650617

2. Li N, Zhu W, Li Y, Gong J, Gu L, Li M, Cao L, Li J. Surgical treatment and perioperative management of idiopathic abdominal cocoon: single-center review of 65 cases. World J Surg 2014;38:1860-7. doi: 10.1007/s00268-0142458-6.

3. Gorsi U, Gupta P, Mandavdhare HS, Singh H, Dutta U, Sharma $\mathrm{V}$. The use of computed tomography in the diagnosis of abdominal cocoon. Clin Imaging 2018;50:171-4. doi: 10.1016/j.clinimag.2018.03.014.

4. Solmaz A, Tokoçin M, Arıcı S, Yiğitbaş H, Yavuz E, Gülçiçek $\mathrm{OB}$ et al. Abdominal cocoon syndrome is a rare cause of mechanical intestinal obstructions: a report of two cases. Am J Case Rep 2015:16:77-80. doi: 10.12659/ AJCR.892658

5. Kashefi AM, Shojaiefard A, Soroush A, Ghorbani AA, Jafari M, Khodadost M, et al. Evaluation of Response to Preoperative Chemotherapy Versus Surgery Alone in Gastroesophageal Cancer: Tumor Resectability, Pathologic Results and Post-Operative Complications. Asian Pac J Cancer Prev 2016;17:231-7. doi: 10.7314/apjcp.2016.17.s3.231

6. Fursevich D, Burt J. Abdominal Cocoon Syndrome: A Rare Cause for Recurrent Abdominal Pain. J Gastrointest Surg 2017;21:1194-5. doi: 10.1007/s11605-017-3358-5.

7. Mandavdhare HS, Kumar A, Sharma V, Rana SS. Abdominal cocoon: an enigmatic entity. Trop Gastroenterol 2016;37:156-67.

8. Sharma V, Mandavdhare HS, Gorsi U, Singh H. Bottle gourd sign: a new radiologic sign of abdominal cocoon on computed tomography. Indian J Gastroenterol 2017;36:SI04.

9. Vlijm A, van Schuppen J, Lamers AB, Struijk DG, Krediet RT. Imaging in encapsulating peritoneal sclerosis. NDT Plus 2011;4:281-4. doi: 10.1093/ndtplus/sfr068.

10. Allam H, Al Yahri O, Mathew S, Darweesh A, Suliman $\mathrm{AN}$, Abdelaziem S, et al. The enigma of primary and secondary encapsulating peritoneal sclerosis. BMC Surg 2016;16:81. doi:10.1186/s12893-016-0198-2
11. Qasaimeh GR, Amarin Z, Rawshdeh BN, El-Radaideh KM Laparoscopic diagnosis and management of an abdominal cocoon: a case report and literature review. Surg Laparosc Endosc Percutan Tech 2010;20:169-71. doi: 10.1097/ SLE.0b013e3181f193ec.

12. Ceulemans LJ, Deferm NP, Deferm S, Willaert RAV, Deferm JT, Vanhoenacker FM. Unusual Cause of Mechanical Ileus: Abdominal Cocoon Syndrome. J Belg Soc Radiol 2016;17;100:36. doi: 10.5334/jbr-btr.1062.

13. Anantha RV, Salvadori MI, Hussein MH, Merritt N. Abdominal cocoon syndrome caused by Mycobacterium bovis from consumption of unpasteurised cow's milk. Lancet Infect Dis 2015;15:1498. doi: 10.1016/S14733099(15)00438-7.

14. Chaudhary P, Kumar R, Ahirwar N, Nabi I, Gautam S, Munjewar C, et al. A retrospective cohort study of 756 cases of abdominal tuberculosis: two decades single centre experience. Indian J Tuberc 2016;63:245-50. doi: 10.1016/j.ijtb.2016.09.010. 